\title{
A SUCCESSFUL COMMUNITY-BASED PILOT PROGRAMME TO CONTROL INSECT VECTORS OF CHAGAS DISEASE IN RURAL GUATEMALA
}

\author{
P. M. PENNINGTON ${ }^{1}$, E. PELLECER RIVERA ${ }^{1}$, \\ S. M. DE URIOSTE-STONE ${ }^{2}$, T. AGUILAR ${ }^{1}$ AND J. G. JUÁREZ ${ }^{1}$ \\ ${ }^{I}$ Center for Health Studies, Universidad del Valle de Guatemala, Guatemala; \\ pamelap@uvg.edu.gt \\ ${ }^{2}$ School of Forest Resources, University of Maine, Orono, Maine, USA
}

\begin{abstract}
SUMMARY
The adoption of novel integrated vector management (IVM) strategies requires proof-of-concept demonstrations. To implement a community-based intervention, for the control of vectors of Chagas disease in Guatemala, we engaged all relevant stakeholder groups. Based on this and previous experiences of the authors on engaged research and community-based interventions, several key factors can help facilitate effective integration of stakeholders in support of area-wide integrated vector management (AWIVM) programmes. First and foremost, the diversity of stakeholders needs to be engaged early-on in the participatory action research and implementation processes, to provide ownership and contribute ideas on how to design and implement an intervention. Another important component, situational analysis regarding current pest control policies, practices and relevant stakeholders, is generated through interviews with key informants, at both national and local levels (governmental and non-governmental organizations); it can facilitate the joint identification of strengths, weaknesses, opportunities and threats regarding current pest control strategies and proposing solutions through an AW-IVM approach. In addition, successful AWIVM can result from identifying locally relevant strategies to implement the proof-of-concept demonstrative project. Further, it is critical to maintain constant communication with the local and national leaders, involving them throughout the implementation and evaluation processes. Flexibility should also be built into the project to allow for community-driven changes in the strategy, through a cyclical joint reflective process. Periodic feedback of project development needs to be scheduled with key stakeholders to maintain rapport. Finally, the results of the evaluation should be shared and discussed with stakeholders to ensure long-term sustainability of the programme, intervention, or project. Here we present the citizen engagement procedures used to integrate community members, health officials, and non-governmental organization staff for Chagas disease control in a region of Guatemala. We demonstrate how these methods can be applied to support AW-IVM programmes, so that communities and authorities are actively involved in the development and implementation of a jointly agreed intervention. In 2012, we developed the IVM intervention in an area of Guatemala with persistent Triatoma dimidiata (Latreille) infestation that is associated with the presence of infected rodents (rats and mice), that act as reservoirs of the Trypanosoma cruzi Chagas parasites inside the households. Nine control communities received only the Ministry of Health insecticide application against the vector and nine intervention communities participated in the
\end{abstract} Development and Field Application, pp. 709-727. CRC Press, Boca Raton, Florida, USA. (C) 2021 IAEA 
AW-IVM intervention. The intervention included a programme for rodent control by the community members, together with education about the risk factors for vector infestation, and insecticide application by the Ministry of Health. Entomological evaluations in 2014 and 2015 showed that vector infestation remained significantly lower in both intervention and control communities. In 2015, we found that there was a higher acceptance of vector surveillance activities in the intervention communities compared to control communities, suggesting that participatory activities increase programme sustainability. Finally, we found that there was a significant increase over time in the number of households with infected vectors in the control group, whereas there was no significant increase in the communities that participated in the programme. Thus, an AW-IVM programme including simultaneous rodent and vector control could reduce the risk of Chagas infection in communities with persistent vector infestation.

Key Words: Central America, Ministry of Health, Jutiapa, community-driven changes, citizen engagement, Reduviidae, Trypanosoma cruzi, Rhodnius prolixus, Triatoma dimidiata, Triatoma infestans, stakeholders, participatory action research, vector surveillance, area-wide integrated vector management (AW-IVM), peridomestic environments, insecticide application, rodent control

\section{BACKGROUND ON CHAGAS DISEASE}

\subsection{Chagas Disease in Latin America}

Chagas disease is widespread in the Americas, affecting 6-7 million people (WHO 2017). It is considered one of the most neglected tropical diseases with serious public health implications, causing the loss of more than 600000 disability-adjusted life years in Latin America (Mathers et al. 2007). The causing agent, Trypanosoma cruzi Chagas, is transmitted primarily by a few species of blood-feeding triatomine insects of the Reduviidae family (Dias et al. 2002). Strategies to control the vector species associated with domestic environments have been successful in several Latin American regions.

There are three area-wide regional initiatives in the Americas for Chagas disease vector control: the Southern Cone, the Andean Pact, and the Central American Initiative (Dias et al. 2002). All three initiatives aim to reduce the incidence of Chagas disease through vertically coordinated multi-country vector control programmes, blood supply screening, and health education (Dias et al. 2002). The two area-wide South American initiatives targeted Triatoma infestans Klug for vector control, as it is the main vector for the transmission of the disease in these regions (Massad 2008). The control activities for $T$. infestans resulted in the interruption of vector-borne transmission in Brazil, Chile and Uruguay (Dias 2007). Also, it reduced the incidence of infection in children and young adults in its member countries (Moncayo and Silveira 2009).

The Central American Initiative focused on the coordinated use of indoor residual spraying (IRS) to eliminate Rhodnius prolixus (Stähl) and reduce Triatoma dimidiata (Latreille) domestic populations (PAHO 2011). This area-wide programme included an attack and a surveillance phase that was coordinated by the Ministers of Health of Central America and several cooperation agencies, including local universities and the Japanese International Cooperation Agency (JICA 2014). 
The success of the IRS interventions resulted in the interruption of Chagas transmission by $R$. prolixus in Guatemala, Honduras and Nicaragua, and the elimination of the vector in Costa Rica, El Salvador and Mexico (Hashimoto and Schofield 2012). These initiatives were successful and led to a significant decrease in the incidence and prevalence of Chagas disease. However, as with many area-wide pest/vector control programmes (Vreysen et al. 2007), remaining foci with persistent infestations hinder regional success. To succeed in these areas will require approaches that integrate novel ecological, biological and social factors.

Since early 2000s, housing improvement was proposed for sustainable control in regions with persistent $T$. dimidiata infestation (Lucero et al. 2013). In 2009, the World Health Organization (WHO) promoted the development of novel interventions for the control of Chagas and dengue in Latin America (Sommerfeld and Kroeger 2015). The interventions included multi-sectoral and -disciplinary ecosystem management strategies. Our study was part of an initiative by several countries to develop novel approaches for the control of T. dimidiata and T. infestans (Gürtler and Yadon 2015). We aimed to develop a community-based strategy for sustainable control of an area with persistent $T$. dimidiata in south-eastern Guatemala (De UriosteStone et al. 2015).

\subsection{Vector Control Programme in Jutiapa, Guatemala}

Guatemala started a major vector control programme in 2000 as part of the National Strategic Plan for Chagas Control (Hashimoto and Schofield 2012). This included several rounds of IRS application in the endemic area that covered over $45000 \mathrm{~km}^{2}$ in the initial and second programme phase (2000-2005) (Hashimoto et al. 2012). In 2009 , Guatemala was certified to have interrupted transmission of the disease by $R$. prolixus, and $T$. dimidiata infestation was reduced nine-fold in the endemic region (Hashimoto and Schofield 2012; Manne et al. 2012). Vector control activities during the 2000-2010 period were estimated to have reduced the number of seropositive school-age children from 5.3\% (1998) to 1.3\% (2005-2006) (Hashimoto et al. 2012). Thus, successful Chagas disease control was achieved in most of the endemic area.

The effectiveness of IRS was evaluated by Hashimoto et al. (2006) across the department of Jutiapa, located in the south-eastern region of Guatemala. In areas where the baseline infestation rates were originally $20 \%$, one spraying cycle reduced infestation to a mean of $1.4 \%$ within 3-21 months (Fig. 1). However, the infestation levels increased to an average of 8.1\% during a second screening 20-45 months after spraying. In villages with an initial $40 \%$ infestation rate, the first spraying cycle reduced it to an average of $12.2 \%$. Given that the control programme aims to reduce infestation to below $5 \%$, a second spraying cycle was carried out in these villages. This effort reduced infestation to $4.8 \%$ in 40 of 52 villages 3-10 months after spraying. However, 12 of the 52 villages showed higher than $5 \%$ infestation after two spraying cycles, necessitating a third cycle that reduced the infestation from $10.9 \%$ to $4.1 \% 3$ 5 months after spraying. 
With uneven results of prevention and control efforts, emphasis shifted to exploring long-term sustainability of surveillance and control interventions (Schofield et al. 2006). Despite a growing recognition of the role of social, cultural, economic and political conditions as risk factors linked to the disease, research and control efforts focusing on these factors have remained scarce (Ventura-García et al. 2013). Vulnerable groups such as indigenous populations and groups living in poverty continue to be at a high risk for disease transmission due to cultural, social, political, and health system barriers (Dell'Arciprete et al. 2014).

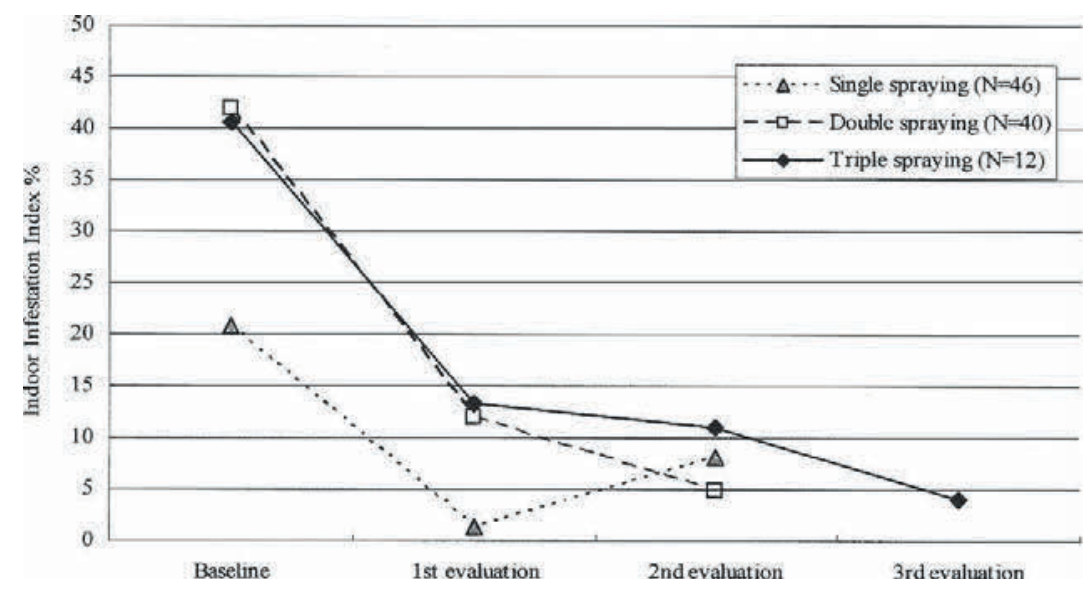

Figure 1. Changes of indoor infestation index for the single, double, and triple spraying areas (from Hashimoto et al. 2006).

\section{DEVELOPMENT OF PROCEDURES AND SURVEYS}

\subsection{Setting the Foundations}

\subsubsection{Area of Interest and Initial Field Visit}

The municipality of Comapa in the department of Jutiapa has been recognized as an endemic focus for Chagas disease, with persistent $T$. dimidiata infestation (Hashimoto et al. 2006) and high prevalence of T. cruzi infection (Rizzo et al. 2003). After multiple cycles of IRS and house improvement interventions (Bustamante et al. 2014), transmission continues at low levels in school-age children (Juárez et al 2018). A multidisciplinary team was formed that was composed of social and biological scientists with the aim to develop a project for improved Chagas disease control in this region. Our aim was to create a process that allowed stakeholders to contribute ideas, and implement an intervention that considered local conditions, resources, and concerns. 


\subsubsection{PRECEDE-PROCEED Framework}

The PRECEDE-PROCEED model (PRECEDE: Predisposing, Reinforcing, and Enabling Causes in Educational Diagnosis and Evaluation; PROCEED: Policy, Regulatory and Organizational Constructs in Educational and Environmental Development) was used as a framework to guide the identification of risk factors through an analysis of the situation, and to develop and implement the intervention programme considering the risk conditions. This framework has been widely used for planning, implementing and evaluating health promotion programmes (Edberg 2007). It uses a multidisciplinary approach that includes disciplines such as sociology, psychology, epidemiology, business, and education (Blank 2006).

The steps required by the process before the intervention - PRECEDE - are based on a situational analysis: social, environmental, entomological, epidemiological, psychological, educational, and institutional assessments. A key component is the identification of risk factors (predisposing and reinforcing), as well as an informed development of the intervention proposal, with input from participants during group meetings. The intervention is based on the PRECEDE findings and implemented through the PROCEED steps in the formative and final evaluation of the intervention. As suggested by Edberg (2007), for the evaluation component, attention needs to be placed on assessing 1) the process of implementation, 2) the impact of the intervention (i.e. changes in knowledge, changes in practices and policies, changes in awareness), and 3) a limited number of outcomes due to the short timeframe of the project.

\subsubsection{Defining the Study Design}

In the PRECEDE stage of the project, a mixed methodology design was used to gain in-depth understanding of social, economic and environmental factors associated with persistent triatomine infestation (Bustamante et al. 2014). The approach allowed for a situational analysis of the community context, as well as for generalization of results and the credibility of the conclusions due to triangulation across research methods (Mertens 2014; Patton 2002).

Each method was selected based on its usefulness to the intervention, framed within the PRECEDE component of the framework. The methodology consisted of five stages: a) building rapport and gaining entry, b) mapping of households and sampling design, c) conducting baseline entomological and household surveys on knowledge, attitudes and practices regarding Chagas disease and possible risk factors, d) facilitating group meetings, and e) analysing documented evidence regarding local Chagas disease control activities and the socio-economic context.

Quantitative and qualitative data analyses allowed jointly developing a situational analysis report and an intervention proposal through a participatory process of individual and group learning and reflection (Bustamante et al. 2014; De UriosteStone et al. 2015). The PROCEED component of the study included a pre-test and post-test control group study design (De Urioste-Stone et al. 2015). Once again, a mixed methodology was used to generate both quantitative and qualitative measures during the intervention (Fig. 2). 


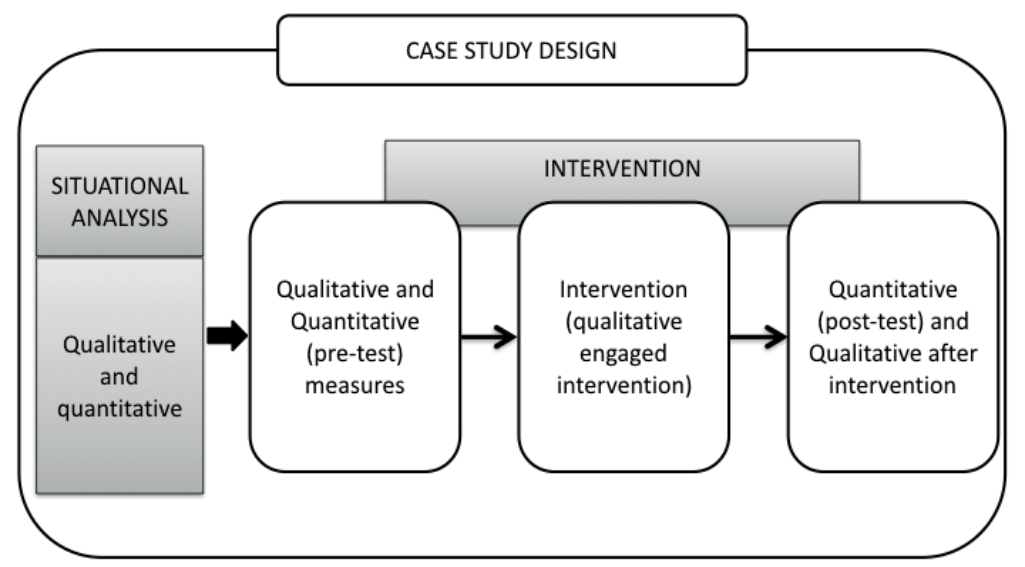

Figure 2. Case study diagram embedded mixed methods and design of the qualitativeengaged intervention (modified from Creswell and Plano Clark 2007).

One-year after completion of the intervention, an interim evaluation was performed in 2014 with household surveys and semi-structured interviews, and twoyears after completion, in 2015, a final entomological survey was performed by the Ministry of Health $(\mathrm{MoH})$ vector control programme. Given that change in behaviour requires time, this stage included pre- and post-surveys as indicators of change in knowledge and attitudes related to identified risk factors and to the disease. This allowed the study to determine the impact of participatory activities on these indicators. Small behavioural changes were measured qualitatively via participatory activities such as giving each participant a calendar to keep track of their activities (e.g. household cleaning and rodent trapping) throughout the month. Entomological indicators at baseline and follow-up were used as a proxy of reduced disease transmission.

\subsubsection{Quality Assurance in Case Study Research}

Trustworthiness strategies (Mertens 2014; Patton 2015) must be applied for quality assurance. Triangulation in case study research is key to enhance credibility (Creswell 1998). We used triangulation across stakeholders (Erlandson et al. 1993; Flick 1998; Mertens 2014; Patton 2015), and by generating information through different research techniques (Erlandson et al. 1993; Flick 1998; Patton 2002; De Urioste-Stone et al. 2015). We validated, through collective and iterative dialogue with the participants, our understanding and interpretation of the main concepts and ideas generated during participatory activities (Creswell 1998; Erlandson et al. 1993; Flick 1998; Mertens 2014). This was done immediately after generating data to enhance credibility. 


\subsubsection{Ethical Considerations of a Multidisciplinary Approach}

A variety of informed consent forms were used; confidentiality was assured by creating coding systems with IDs (questionnaires, interviews, and group meetings), and careful and secure data management. Written consent forms were read and signed by the participants; in case the person could not sign the form, a fingerprint was requested, as well as a signature of a witness. For group activities, a written consent form was used; the consent form was read and signed by a representative of the group and a member of the research team (and included a list of the participants). Participants were requested to sign a consent form for photographs and videos during participatory group meetings. Potential benefits of the study were described and agreed upon prior to data collection.

The consent protocols for the cognitive study were collectively developed with the communities during the ethnographic phase, taking as model the consent forms used for interviews and signing a letter of conformity with the communities according to their own terms. We also were flexible in changing procedures and techniques to respond to contextual particularities in the communities of study. For example, the informed consent originally proposed was a verbal consent, but the communities requested to sign them during the first pilot study, so change to a written consent form was approved by the ethics committee.

After facilitation of each meeting, the research team went through a reflective process and preliminary analysis of results before planning the next group session. Hence, formative data analysis informed data collection.

\subsection{Gaining Entry and Building Rapport}

Gaining entry and building rapport are essential when conducting qualitative research (Ely et al. 1991). We considered this element of the research process essential for carrying out any type of intervention, and to ensure active collaboration of participants. Early on, a meeting was called by the leader of the Community Development Councils (COCODEs) for the entire community to share the study objectives, methods and expected participation at the community and individual level. For participatory activities, the entire community was invited.

For surveys and intervention phases, communities were randomly selected, and a set number of households was randomly selected to be included in the activities. The selection process was explained at these meetings to prevent any misunderstanding.

The following activities were carried out to enhance the success in gaining access and building trust with stakeholders in the study area:

- Periodic communication with gatekeepers from organizations and communities.

- Meetings in Comapa to present the intervention strategies and results from each stage of the project to local leaders, communities, participants and other stakeholders. In every meeting there was a space for dialogue to obtain feedback about concerns and ideas.

- Rapport with the national authorities was also enhanced, and the interim and final results were presented annually at the National Chagas Vector Control Programme evaluation meetings. 
- Presentation of results from each stage of the study to communities and stakeholders.

- Flexibility to change procedures and techniques to respond to the situations, getting Institutional Review Board approval for protocol modifications as the project progressed.

- Respect and value for the time provided by co-participants according to their cultural norms and conception of time.

- Recognition, adaptation and respect for organizational and ethnic cultures and ways.

- Collaborative definition of the location, dates, and times to conduct meetings, interviews and workshops.

- Ongoing reflection on the processes of gaining entry and building trust.

Community meetings were conducted to share general results of the Knowledge, Attitude and Practices (KAP) questionnaires and entomological surveys after the PRECEDE and PROCEED stages. Interim and final results were presented annually at the National Chagas Vector Control Programme evaluation meetings.

\subsubsection{Engaging National and Local Health Authorities}

Before preparing the proposal, the idea was first presented to the head of the National Chagas Vector Control Programme. After approval at the national level, the idea was presented to the Jutiapa Health Area epidemiologist. During this meeting, a visit to the field site provided context and shaped the proposal for local relevance. This visit was critical to gain support from the local health authorities, who provided valuable input for the final proposal. Brainstorming sessions looked at potential stakeholders to approach, and potential collaboration activities and resources. Meetings were also conducted with leaders from the Municipality of Comapa. The main objective of these meetings was to gain stakeholder permission to conduct the research, to explain its different components and to discuss and gain feedback on how to implement them. Local authorities provided us with baseline data from the communities, maps, and an up-dated list of community leaders.

\subsubsection{Engagement of Community Leaders}

In 2002, the COCODEs were created in Guatemala to serve as the local organization that identifies and brings together community leaders. The aim of the COCODEs is to serve as a channel to facilitate the participation of the population, to plan and implement development efforts using a democratic approach (Congreso de la República de Guatemala 2002). COCODEs have usually facilitated introduction of projects (or any external initiative) to their communities, serving as the first contact to approach the community and gain access and project approval.

For our project, a list of communities and their leaders was generated at the municipality level. A meeting was organized with 74 members of the COCODEs from Comapa, where the leaders were informed about the duration and objectives of the project. The methodology was explained in detail, as well as the expected participation of the community members. Lists of problems related to Chagas and organizations working in the area were generated and the overall scope of knowledge 
and experiences with Chagas at the community level was detailed. The meeting with the COCODEs leaders was essential to gaining entry and ensure proper communication about the project.

However, the COCODEs are also an institution that can influence decisionmaking, which may lead to politicization of initiatives. New projects with no former experience in the area should pay attention to these political dynamics, which might be very challenging. In our case, some communities had one COCODE appointed by the municipality and another appointed by the community members. At the end two meetings were done for each community. We found throughout the study that identifying leaders who were recognized by the community allowed us to gain entry and build a relationship of trust with each community, without politicizing the project. In the absence of organizations such as COCODE, one option is to determine which other institutions collaborate with local leaders and which organizations (governmental and non-governmental) are working in the area. It is very likely that relevant structures are already in place, i.e. a network of leaders, youth teams, volunteers or women groups, that could be invited to collaborate, rather than starting interactions from scratch.

\subsubsection{Community Engagement}

Based on our experience, securing collaboration from the communities is essential to avoid a paternalistic approach. It was important to acknowledge that we did not have all the answers and that we could not propose all potential solutions using solely ideas generated in the office or by conducting experiments in the laboratory. We recognized the need to listen to the local community members, and value their opinions to make the intervention successful and sustainable.

After evaluating the eco-bio-social baseline information, we conducted participatory activities at three different communities, inviting all members of each community to each meeting through the local leader. The communities were selected based on different social organization characteristics and vector infestation levels. In these meetings, we actively listened to the ideas and concerns of local participants regarding their role in risk factor mitigation.

Based on this approach, we proposed potential strategies that were discussed with all stakeholders and could be modified later if required. Actively listening to ideas, experiences and respecting the knowledge of local populations was key to engaging and empowering participants. Through this engagement, the project moved from being an endeavour from a traditional research team, to becoming a collective undertaking with the active participation of local populations - this is essential for the activities to continue after the project has come to an end.

\subsection{Stakeholders Analysis}

\subsubsection{Stakeholder Context}

We developed a list of stakeholders at the national, regional and local levels that stipulated their roles in Chagas vector control. The different roles and areas of emphasis for stakeholders are shown in Fig. 3. 


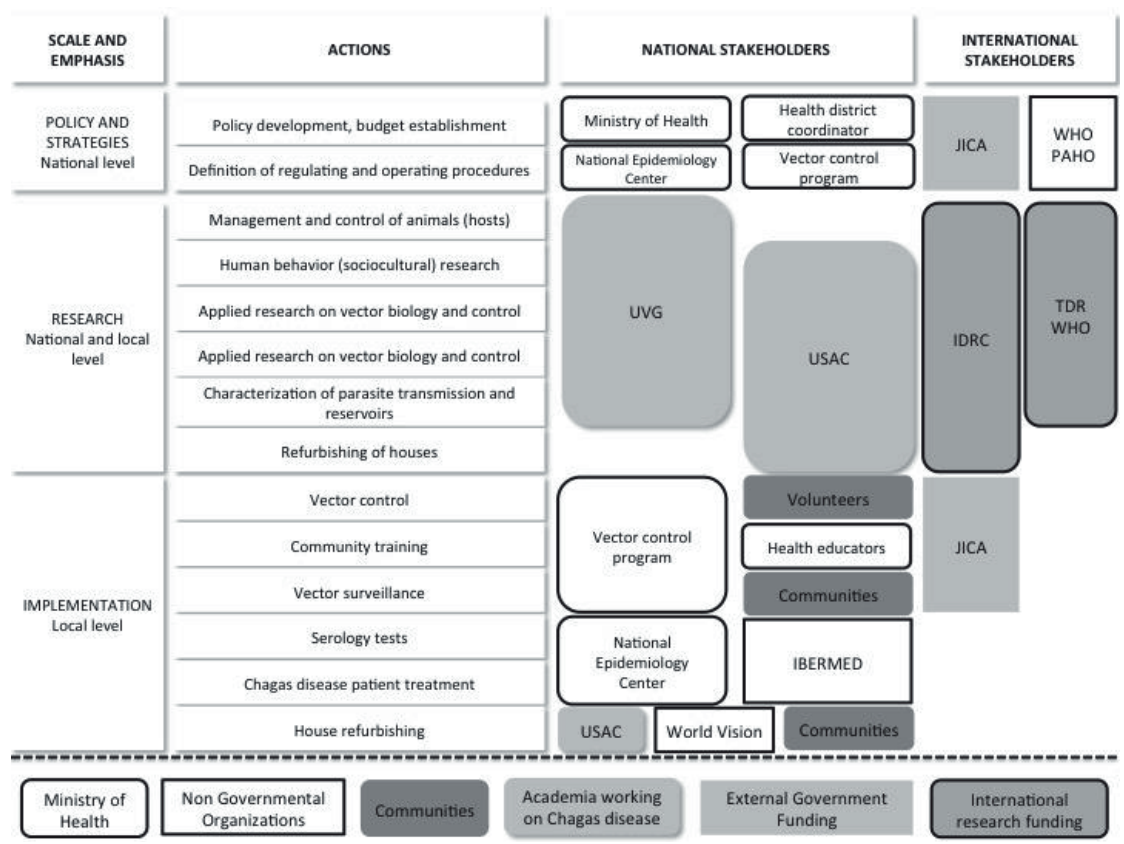

Figure 3. Stakeholder mapping of the National System for Chagas Control at the local and national levels (with permission from De Urioste-Stone et al. 2015). (Universidad del Valle de Guatemala: UVG; Universidad de San Carlos de Guatemala: USAC; International Development Research Centre: IDRC; Japan International Cooperation Agency (JICA); Tropical Disease Research-WHO: TDR-WHO; Pan American Health Organization: PAHO; Médicos con Iberoamérica: IBERMED).

The relationships we observed between the different stakeholders are portrayed in Fig. 4. This type of analysis is useful to understand and identify the persons who can positively contribute to the project and those who can potentially be obstructive. During the study, we continuously discussed newly developing power relationships between the different stakeholders to predict potential conflicts, but also opportunities for collaboration and to leverage resources.

We strived to understand the different roles and interactions among stakeholders to build the relationships for a participatory process. We acknowledged and considered knowledge about Chagas disease and its control, including interests, positions, alliances and relevance in Chagas disease control of those involved (Schmeer 1999).

\subsubsection{Training of Personnel/Collaborators}

Several training sessions were undertaken with local field staff of the MoH. The first efforts focused on standard operating procedures, use of maps and global positioning system (GPS) equipment. Field staff received training on how to apply the household/KAP questionnaire, specifically on how to approach interviewees, how to ask each of the questions, how to facilitate consent request and other ethical concerns. 
Training sessions on the biosafety and handling of vector specimens were undertaken by all members of the vector control $\mathrm{MoH}$ team. These activities were crucial to generate understanding and empowerment related to the proposed activities for the intervention; all staff and collaborators need to fully understand the procedures to generate high quality data. This approach also allowed for collaboration across disciplines and forming a strong interdisciplinary team.

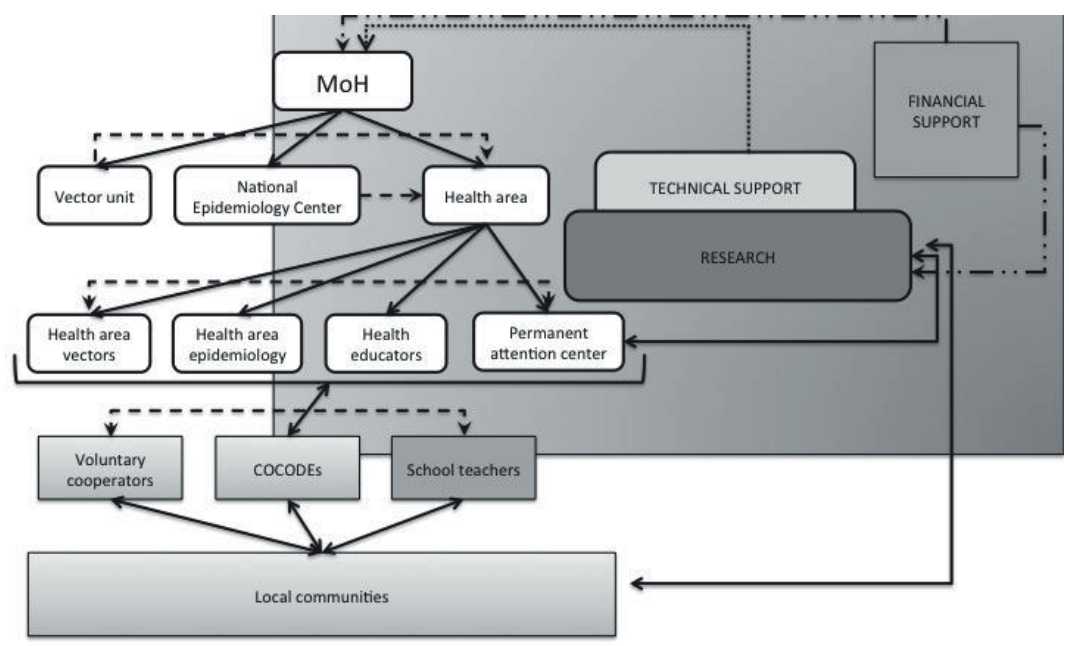

Figure 4. Mapping of stakeholder relationships. Arrows denote direction of relationships between stakeholders: unidirectional denoting supervision relationships, bidirectional being collaborative interactions. The dotted lines indicate secondary linkages through cooperation relationships, the solid lines indicate primary linkages through collaborative relationships.

\subsubsection{Pre-testing of Survey Procedures and Questionnaire}

We validated all survey procedures, questionnaires and the consent forms before starting data collection, in two communities from the same municipality that were not part of our study. These communities were selected from the sample of communities found over 850 meters above sea level that were not selected for the survey. Two validation rounds were conducted in two different communities with four households randomly selected in each. We obtained feedback to make the required modifications.

Each household was visited to assess the household/KAP and entomological forms, with the goal of reducing measurement error (Dillman et al. 2008; Krosnick et al. 2014). The reactions of interviewed community members to wording and ordering of questions was observed and analysed, and participants were asked to suggest better phrasing and more appropriate wording when questions were not clear. As a result, the accuracy of the questions was improved, several questions were added, and the order of questions revised. The order of the procedures was also modified to improve the interviewing process by performing the KAP survey instrument before the entomological survey. 
The instruments were modified, and a third round took place to pilot test the effectiveness of the selected protocols, with four more households randomly selected. After the feedback from the pilot test was included, the household/KAP questionnaires were further reviewed and revised by staff of the $\mathrm{MoH}$.

\section{THE INTERVENTION}

\subsection{Developing the Intervention}

Before the intervention, three communities of the Municipality of Comapa, selected based on their vector infestation levels (two with the highest infestation and one without persistent infestation), were invited to share information regarding animal management in relation to Chagas disease risk factors and to identify their problems and potential solutions (Bustamante et al. 2014). All community members were invited to these activities. We used a participatory model to gain in-depth understanding regarding local practices related to risk factors. Through a series of community reflection exercises, it became evident that community members wanted to better understand the disease, and to identify actions to change the current conditions.

An anthropological study was undertaken to better understand the economic production practices, further adding to the knowledge generated previously. We observed very distinct activities based on gender, with agricultural activities mainly carried out by men, whereas women carried out household chores, raised children, cared for peridomestic animals (e.g. chickens), and traded goods with other community members.

The first evaluation of the region showed that, with respect to Chagas disease, chicken management practices and the presence of dogs and rodents posed an important risk for the household (Bustamante et al. 2014). The findings indicated the importance of developing sustainable animal and environmental management practices that would modify behaviours of community members that posed risk factors in relation to triatomine infestation, as part of a gender-oriented education programme.

\subsection{The Intervention Framework}

The intervention framework included a close collaboration with the communities to generate an integral animal and environmental management programme. It also aimed to implement relevant components of another educational programme in the area called "Clean House, Clean Patio" developed by the MoH and JICA (De UriosteStone et al. 2015). The general objective was to provide a strategy to improve Chagas disease prevention in different ecological and social settings. This was achieved by better understanding the ecological, vector-biological and social ("eco-bio-social") determinants of peridomestic animal management in relation to vector infestation. As a result, we developed and evaluated a community-centred intervention to reduce habitats for rodents and chickens inside the household, both of which were found to be important blood sources and risk factors for triatomines. 
Eighteen communities with baseline infestation levels above $15 \%$ were selected from the 30 communities surveyed in the situational analysis (De Urioste-Stone et al. 2015). Nine communities were randomly assigned to the intervention (AW-IVM) and the other nine communities to the control (only the $\mathrm{MoH}$ insecticide application) groups (Fig. 5).

\subsection{Participation and Community Engagement during Intervention}

We used some elements of Participatory Action Research (PAR) to guide changes in perception and behaviour at multiple levels, through "direct involvement, intervention or insertion in processes of social action" (Fals-Borda 2001). The model facilitated active listening to the concerns and ideas of participants, and it promoted a reflexive process about the implications of the interventions amongst researchers and participants. We relied on an iterative process of reflection and action (Freire 1970).

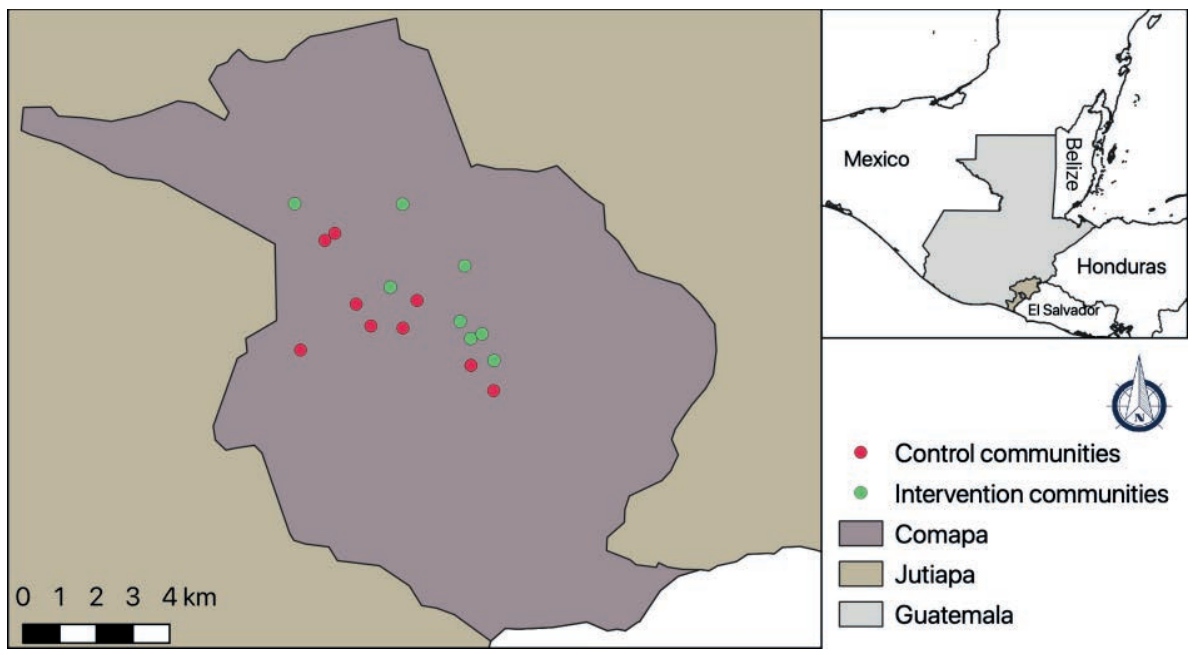

Figure 5. Distribution of communities assigned to intervention and control groups for the intervention phase, Comapa, Jutiapa, Guatemala, 2012.

We - participants, researchers and collaborating institutions (non-governmental organizations, $\mathrm{MoH}$ ) - engaged in a cycle of sharing knowledge, following up changes in practices, and reflecting about the research process during the intervention (De Urioste et al. 2015). We believe that through this knowledge-reflection-action iterative method, we facilitated a process best described by Kemmis and McTaggart (2003) as:

"...systematic inquiry, process; participation and collaboration; bridging local and scientific knowledge; empowerment; and action”. 
Participatory techniques (FAO 1990; Chambers 2002) were used as a vehicle to stimulate dialogue and reflection among participants about Chagas disease. Educational material about Chagas and topics related to chicken, dog, rodent/grain management were discussed and analysed with participants. Seven monthly participatory meetings were held within each of the intervention communities:

- Meeting 1: A full description of the intervention methodology and timeline was presented to the selected members of the intervention communities. Consent of all the participants was obtained. Methods were discussed and feedback of the participants was incorporated into the research tools to be developed in the following meetings. An informative brochure that contained results obtained in the baseline was developed and shared with the participants.

- Meeting 2: A community level Strengths, Weaknesses, Opportunities, Threats (SWOT) analysis was generated, to have a better understanding of the situation and to identify specific issues to be addressed in the following meetings. The analysis included topics such as (1) current practices to manage and control the triatomines, (2) current knowledge about $T$. dimidiata behaviour and presence in the houses, and (3) other institutions working directly or indirectly with the disease and the vector.

- Meeting 3: Group narrative related to knowledge and experience with Chagas disease. The research team presented information on the disease and its vector, forms of transmission, symptoms, effects and treatment. A calendar was presented and validated as a personal matrix to document household activities.

- Meeting 4: Group narrative related to knowledge regarding rodents and grain storage as risk factors for vector presence in the house. The research team presented information on the biology and ecology of rodents, the danger they pose in relation to Chagas disease, and proposals for control strategies. The calendars were delivered to the meeting participants for use as personal matrices to record the activities and practices related to rodent and grain storage, to be implemented during that month. During this meeting, rodent traps were delivered, and a practical session demonstrated the use of the traps, protocols to kill the rodents easily and ethically, and to manage and bury the carcasses.

- Meeting 5: Group evaluation with respect to the dynamics of the PAR process, i.e. assessment of the knowledge gained on the vector, the disease and the rodents, the activities proposed for rodent control, and about the use of the matrix (a calendar) to document rodent and grain storage practices. Discussion of needed changes in design and new personal commitments acquired to continue advancing.

- Meeting 6: Group discussion on the use of the matrix for rodent control activities and any related changes made. Sharing of knowledge and experiences related to chickens, vegetation and waste management as risk factors for vector presence. Presentation by the research team on the importance of chicken management, and proposal of a strategy for integral waste management, through compost production, a chicken coop and food production. Validation and discussion about the matrices to have a record of the activities and practices related to environmental management. 
- Meeting 7: Group discussion regarding the use of the matrix for grain storage, and rodent, chicken, dog and environmental management practices and any changes made, and all steps of the PAR process. Closing presentation with findings, achievements and identification of possible knowledge gaps. Opinion survey among participants and local leaders regarding the PAR process.

\section{RESULTS AND CONCLUDING REMARKS}

Comparison of KAP survey results with Student's t test, in a pre-test in 2012 and post-test in 2014, showed that the intervention with participatory activities produced a significant change in protective practices against risk factors for persistent $T$. dimidiata infestation, including rodent control using mechanical traps and environmental management, as well as chicken management (Student's t test, $\mathrm{p}<0.001$ ) (De Urioste-Stone 2015). An odds ratio comparison of entomological indices in 2012 and 2014 showed higher early instar reinfestations in the communities that received no treatment (control), compared to the intervention communities (OR 8.3, 95\% CI 2.4-28.4). In addition, there was a significant reduction in rodent infestations in the intervention group over time (OR 1.9, 95\% CI 1.09-3.45). The overall infestation levels were maintained below 10\% in both intervention and control groups during the first evaluation in 2014 (Fig. 6).

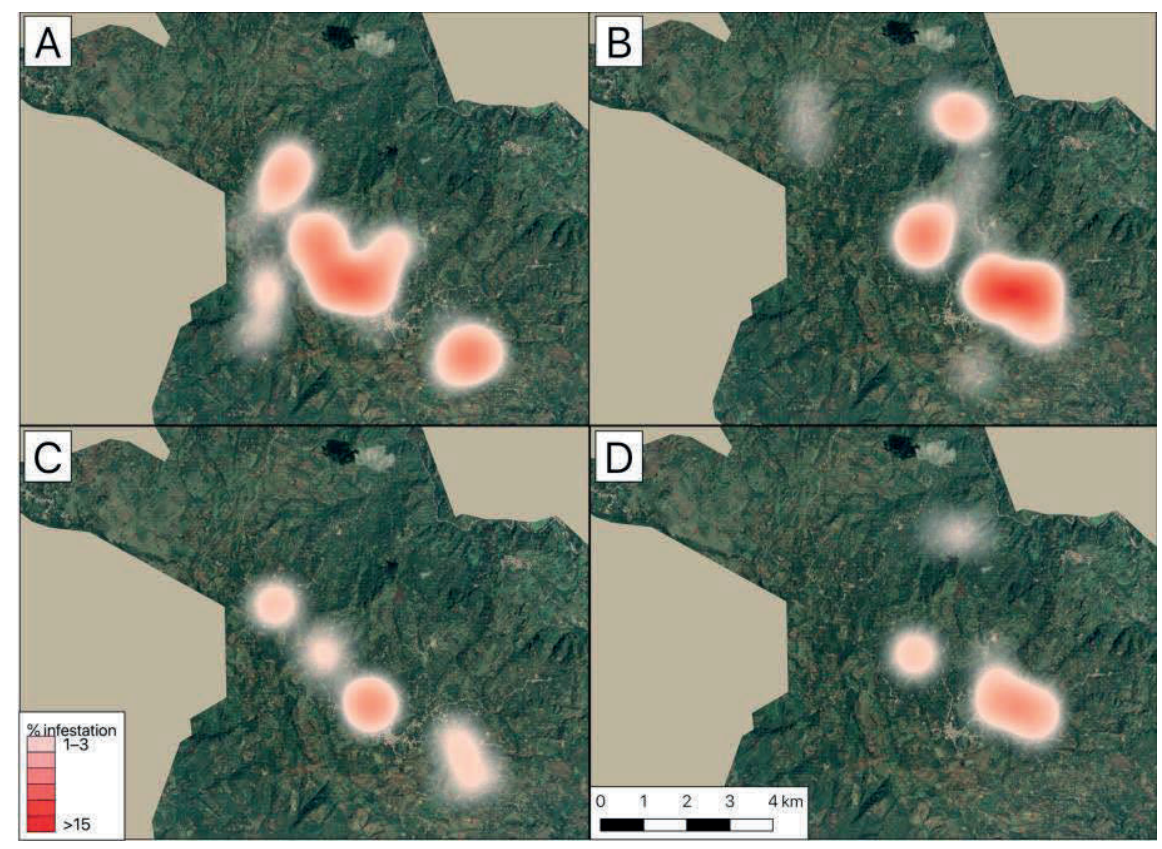

Figure 6. Reduction of Triatoma dimidiata infestation in the Municipality of Comapa, Department of Jutiapa, Guatemala in the control and intervention groups pre- (2012) and post-intervention (2014). A) Control group 2012. B) Intervention group 2012. C) Control group 2014. D) Intervention group 2014. 
In 2015, an entomological survey was performed as part of the $\mathrm{MoH}$ surveillance activities. The survey was performed simultaneously with a serological survey of household inhabitants (Juarez et al 2018). During this 2015 survey, we observed that communities where Participatory Action Research was conducted had a higher participant retention, when comparing treatments (Pearson $\mathrm{Chi}^{2}=3.298, \mathrm{p}=0.046$, one-tailed test). A trend was also observed, at a $90 \% \mathrm{CI}$, for lower drop-out rates for the intervention communities with an Odds Ratio of $1.67(90 \% \mathrm{CI}=1.05,2.65)$ (Table 1). This suggests that the participatory process increases long-term community acceptance of $\mathrm{MoH}$ surveillance activities.

Table 1. Community recruitment and continued participation between control and intervention treatments in numbers (\%) for the municipality of Comapa, Department of Jutiapa, Guatemala

\begin{tabular}{|c|c|c|c|c|c|}
\hline & 2012 & 2014 & 2014 & 2015 & 2015 \\
\hline Treatment & Recruitment & Drop-out & Remained & Drop-out & Remained \\
\hline Control & $215(49.9)$ & $15(3.5)$ & $200(46.4)$ & $37(8.6)$ & $178(41.3)^{*}$ \\
\hline Intervention & $216(50.1)$ & $23(5.3)$ & $193(44.8)$ & $24(5.6)$ & $192(44.5)^{*}$ \\
\hline Total & $431(100)$ & $38(8.8)$ & $393(91.2)$ & $61(14.2)$ & $370(85.8)$ \\
\hline
\end{tabular}

* Significant difference, $p<0.05$. We were unable to observe any statistical difference for domestic infestation levels between the control $(21 \% ; 8.5 \%$ and $15 \%)$ and intervention $(20 \% ; 8.3 \%$ and $12 \%)$ by year (2012-2014-2015). We did observe a trend that may suggest that intervention practices may prevent long-term reinfestation of the houses $(n=192)$ that were originally infested (OR 2.5, 95\% CI 0.93-6.99). On the other hand, the control group ( $n=178)$ showed a higher probability of finding infestation if the household was previously infested (OR 3.16, 95\% CI 1.1-9.00)

We also evaluated the effects of rodent infestation on vector infection by $T$. cruzi, for the triatomines collected in 2012 and 2015. Triatomine infections were confirmed using PCR for the parasite T. cruzi (Maddren 2018). We found that the proportion of infested houses with infected triatomines significantly increased over time in the control group (Table 2). However, in the intervention group the proportion of infested houses with infected triatomines did not increase over time. This suggests that rodent control may reduce the risk of infection in the households. It appears that the participatory process gave the household inhabitants the tools to reduce risk factors for infection. Through this community-based programme, we learned that complex health problems such as vector-borne zoonotic diseases require multidisciplinary and community-based approaches to develop innovative solutions that target ecological, socio-economic, cultural, institutional, and biophysical factors of risk. 
To develop relevant solutions, an in-depth understanding of the dynamics of communities and the role/interactions among stakeholders are first needed to be able to understand the context that frames the public health issue. Once biophysical and social science data are collected and triangulated, and the problems are identified based on this data integration, solutions should include the input from those that will be involved and affected by the intervention.

Table 2. Percentage of sampled houses in the Municipality of Comapa with Triatoma dimidiata infected with Trypanosoma cruzi

\begin{tabular}{|c|c|c|c|}
\hline Treatment & Year & $\begin{array}{c}\text { Infested } \\
\text { Houses }\end{array}$ & $\begin{array}{l}\text { Percent of houses with T. cruzi } \\
\text { infected T. dimidiata }(95 \% \text { CI) }\end{array}$ \\
\hline Control & 2012 & 30 & $65.1(50.3-79.8)^{*}$ \\
\hline Intervention & 2012 & 18 & $90.4(78.2-100.0)^{*}$ \\
\hline & 2015 & 17 & $68.1(44.2-91.9)$ \\
\hline
\end{tabular}

* Significant difference, $C h i^{2}=5.8, p<0.05$

A participatory-reflexive process can help improve the effectiveness of strategies aimed at achieving changes in human social and behavioural contexts. These activities empower the community to engage in practices that are truly relevant and feasible to them. Introduction of AW-IVM innovations should engage citizens throughout the process to ensure public awareness and involvement in the projects.

In our case, given that these vectors can colonize peridomestic environments, it will be necessary to implement additional strategies in the future to reduce the availability of these habitats and prevent future domestic infestations. In addition, all members of the communities should be engaged in the process, to ensure that peridomestic environments do not become a source of infestation for neighbouring households and communities.

\section{REFERENCES}

Blank, M. E. 2006. El modelo precede/proceed: Un organizador avanzado para la reconceptualización del proceso de enseñanza-aprendizaje en educación y promoción de la salud. Salus Online 10: 18-25.

Bustamante, D. M., S. M. De Urioste-Stone, J. G. Juárez, and P. M. Pennington. 2014. Ecological, social and biological risk factors for continued Trypanosoma cruzi transmission by Triatoma dimidiata in Guatemala. PLoS One 9(8): e104599.

Chambers, R. 2002. Participatory workshops: A sourcebook of 21 sets of ideas and activities. Earthscan, London, UK. 236 pp.

Congreso de la República de Guatemala. 2002. Ley de los Consejos de Desarrollo Urbano y Rural. Decreto Numero 11-2002 del Congreso. Ciudad de Guatemala, Guatemala.

Creswell, J. W. 1998. Qualitative inquiry and research design, choosing among five traditions. SAGE Publications, Thousand Oaks, California, USA.

Creswell, J. W., and V. L. Plano Clark. 2007. Designing and conducting mixed methods research. SAGE Publications, Thousand Oaks, California, USA. 
Dell'Arciprete, A., J. Braunstein, C. Touris, G. Dinardi, I. Llovet, and S. Sosa-Estani. 2014. Cultural barriers to effective communication between indigenous communities and health care providers in Northern Argentina: An anthropological contribution to Chagas disease prevention and control. International Journal for Equity in Health 13: 6.

De Urioste-Stone, S. M., P. M. Pennington, E. Pellecer, T. M. Aguilar, G. Samayoa, H. D. Perdomo, and J. G. Juárez. 2015. Development of a community-based intervention for the control of Chagas disease based on peridomestic animal management: An eco-bio-social perspective. Transactions of the Royal Society of Tropical Medicine and Hygiene 109: 159-167.

Dias, J. C. P. 2007. Southern Cone Initiative for the elimination of domestic populations of Triatoma infestans and the interruption of transfusion Chagas disease: Historical aspects, present situation, and perspectives. Memórias do Instituto Oswaldo Cruz 102 (Suppl. 1): 11-18. Epub August 31, 2007.

Dias, J. C. P., A. C. Silveira, and C. J. Schofield. 2002. The impact of Chagas disease control in Latin America: A review. Memórias do Instituto Oswaldo Cruz 97: 603-612.

Dillman, D., J. D. Smyth, and L. M. Christian. 2008. Internet, mail, and mixed-mode surveys: The tailored design method. John Wiley \& Sons, Inc., Hoboken, New Jersey, USA.

Edberg, M. 2007. Essentials of health behavior: Social and behavioral theory in public health. Jones \& Bartlett Publishers, Sudbury, Massachusetts, USA.

Ely, M., M. Anzul. T. Friedman, D. Garner, and A. McCormack Steinmetz. 1991. Doing qualitative research: Circles within circles. The Falmer Press, London, UK.

Erlandson, D. A., E. L. Harris, B. L. Skipper, and S. D. Allen. 1993. During naturalistic inquiry: A guide to methods. SAGE Publications, Newbury Park, California, USA.

(FAO) Food and Agriculture Organization of the United Nations. 1990. The Community's toolbox: The idea, methods and tools for participatory assessment, monitoring and evaluation in community forestry. FAO Regional Wood Energy Development Programme in Asia, Bangkok, Thailand.

Fals-Borda, O. 2001. Participatory (action) research in social theory: Origins and challenges, pp. 27-37. In P. Reason and H. Bradbury (eds.), Handbook of action research: Participative inquiry and practice. SAGE Publications, Thousand Oaks, California, USA.

Flick, U. 1998. An introduction to qualitative research. London: SAGE Publications, Thousand Oaks, California, USA.

Freire, P. 1970. Pedagogy of the oppressed. Herder and Herder. New York, New York, USA. 186 pp.

Gürtler, R. E., and Z. E. Yadon. 2015. Eco-bio-social research on community-based approaches for Chagas disease vector control in Latin America. Transactions of the Royal Society of Tropical Medicine and Hygiene 109: 91-98.

Hashimoto, K., and C. J. Schofield. 2012. Elimination of Rhodnius prolixus in Central America. Parasites \& Vectors 5: 45.

Hashimoto, K., C. Cordon-Rosales, R. Trampe, and M. Kawabata. 2006. Impact of single and multiple residual sprayings of pyrethroid insecticides against Triatoma dimidiata (Reduviiade; Triatominae), the principal vector of Chagas disease in Jutiapa, Guatemala. The American Journal of Tropical Medicine and Hygiene 75: 226-230.

Hashimoto, K., H. Alvarez, J. Nakagawa, J. Juarez, C. Monroy, C. Cordon-Rosales, and E. Gil. 2012. Vector control intervention towards interruption of transmission of Chagas disease by Rhodnius prolixus, main vector in Guatemala. Memórias do Instituto Oswaldo Cruz 107: 877-887.

(JICA) Japan International Cooperation Agency. 2014. Summary best practices from the Chagas disease control in Guatemala, El Salvador, Honduras and Nicaragua. 33 pp.

Juárez, J. G., P. M. Pennington, J. P. Bryan, R. E. Klein, C. B. Beard, E. Berganza, N. Rizzo, and C. Cordon-Rosales. 2018. A decade of vector control activities: Progress and limitations of Chagas disease prevention in a region of Guatemala with persistent Triatoma dimidiata infestation. PLoS Neglected Tropical Diseases 12(11): e0006896.

Kemmis, S. and R. McTaggart. 2003. Participatory action research, pp. 336-396. In N. K. Denzin and Y. S. Lincoln (eds.), Strategies of qualitative inquiry. SAGE Publications, Thousand Oaks, California, USA.

Krosnick, J. A., P. J. Lavrakas, and N. Kim. 2014. Survey research, pp. 404-442. In H. T. Reis and C. M. Judd (eds.), Handbook of research methods in social and personality psychology. Second Edition. Cambridge University Press, Cambridge, UK.

Lucero, D. E., L. A. Morrissey, D. M. Rizzo, A. Rodas, R. Garnica, L. Stevens, D. M. Bustamante, and M.C. Monroy. 2013. Ecohealth interventions limit triatomine reinfestation following insecticide spraying in La Brea, Guatemala. The American Journal of Tropical Medicine and Hygiene 88: 630 637. 
Maddren, R. 2018. Characterisation of domestic T. cruzi transmission in South-Eastern Guatemala: Genetic analysis of the major vector species Triatoma dimidiate. Master thesis, London School of Hygiene and Tropical Medicine, London, UK.

Manne, J., J. Nakagawa, Y. Yamagata, A. Goehler, J. S. Brownstein, and M. C. Castro. 2012. Triatomine infestation in Guatemala: Spatial assessment after two rounds of vector control. The American Journal of Tropical Medicine and Hygiene 86: 446-454.

Massad, E. 2008. The elimination of Chagas' disease from Brazil. Epidemiology and Infection 136: $1153-$ 1164.

Mathers, C. D., M. Ezzati, and A. D. Lopez. 2007. Measuring the burden of neglected tropical diseases: The global burden of disease framework. PLoS Neglected Tropical Diseases 1(2): e114.

Mertens, D. M. 2014. Research and evaluation in education and psychology: Integrating diversity with quantitative, qualitative, and mixed methods (4th ed.). SAGE Publications, Thousand Oaks, California, USA.

Moncayo, Á., and A. C. Silveira. 2009. Current epidemiological trends for Chagas disease in Latin America and future challenges in epidemiology, surveillance and health policy. Memórias do Instituto Oswaldo Cruz 104 (Suppl. 1): 17-30.

(PAHO) Pan American Health Organization. 2011. Iniciativa de los países de América Central para la interrupción de la transmisión vectorial y transfusional de la enfermedad de Chagas (IPCA): Historia de 12 años de una iniciativa subregional 1998-2010. Representación de la Organización Panamericana/Mundial de la Salud en Honduras. Tegucigalpa, Honduras. 89 pp.

Patton, M. Q. 2002. Qualitative research \& evaluative methods (3rd ed.). SAGE Publications, Thousand Oaks, California, USA.

Patton, M. Q. 2015. Qualitative research \& evaluative methods: Integrating theory and practice (4th ed.). SAGE Publications, Thousand Oaks, California, USA.

Rizzo, N. R., B. A. Arana, A. Diaz, C. Cordon-Rosales, R. E. Klein, and M. R. Powell. 2003. Seroprevalence of Trypanosoma cruzi infection among school-age children in the endemic area of Guatemala. American Journal of Tropical Medicine and Hygiene 68: 678-682.

Schmeer, K. 1999. Guidelines for conducting a stakeholder analysis. Partnerships for Health Reform, Abt Associates Inc. Bethesda, Maryland, USA.

Schofield, C. J., J. Jannin, and R. Salvatella. 2006. The future of Chagas disease control. Trends in Parasitology 22: 583-588.

Sommerfeld, J. and A. Kroeger. 2015. Innovative community-based vector control interventions for improved dengue and Chagas disease prevention in Latin America: Introduction to the special issue. Transactions of The Royal Society of Tropical Medicine and Hygiene 109 (2): 85-88.

Ventura-García, L., M. Roura, C. Pell, E. Posada, J. Gascón, E. Aldasoro, J. Muñoz, and R. Pool. 2013. Socio-cultural aspects of Chagas disease: A systematic review of qualitative research. PLoS Neglected Tropical Diseases 7(9): 1-8.

Vreysen, M. J. B., A. S. Robinson, and J. Hendrichs (eds.). 2007. Area-wide control of insect pests: From research to field implementation. Springer Dordrecht, The Netherlands. $789 \mathrm{pp}$.

(WHO) World Health Organization. 2017. Fact sheet: Chagas disease (American trypanosomiasis). Washington, DC, USA. 